\title{
Mineração
}

\section{Teoria de Kubelka-Munk aplicada na indústria de minerais industriais: predição do teor de contaminantes em caolim}

\author{
(Kubelka-Munk theory applied to industrial minerals: \\ prediction of impurity content in kaolin)
}

\author{
Ítalo Gomes Gonçalves \\ Graduando em Engenharia de Minas - UFRGS - Porto Alegre - RS - Brasil \\ E-mail: italogoncalves@ibest.com.br \\ Carlos O. Petter \\ Professor do Departamento de Engenharia de Minas - UFRGS - Porto Alegre - RS - Brasil \\ E-mail: cpetter@ufrgs.br
}

\section{Resumo}

Para a maioria dos minerais industriais, o valor econômico do produto está associado, diretamente, às suas propriedades óticas, como cor, alvura, etc. Essas propriedades óticas também podem ser utilizadas como ferramenta auxiliar no planejamento de lavra e processamento do minério. Nesse trabalho, a teoria de Kubelka-Munk foi utilizada na tentativa de criar um método para a determinação da concentração de contaminantes em caolim. Devido a dificuldades práticas, foi necessário o desenvolvimento de algumas relações matemáticas envolvendo o modelo para a obtenção das duas constantes necessárias para cada material. Os resultados obtidos foram satisfatórios, possibilitando a realização de estudos mais detalhados sobre a aplicação dessas relações matemáticas, tanto para o caolim, como para os minerais industriais em geral.

Palavras-chave: Alvura, caolim, Kubleka-Munk, contaminantes.

\begin{abstract}
For most industrial minerals, the economic value of the product is directly associated to its optical properties, like color, brightness, etc. These optical properties can also be used as auxiliary tools in the mine planning and mineral processing. In this work, the Kubelka-Munk Theory was used in an attempt to create a method for the determination of contaminant concentration in kaolin. Due to practical difficulties, it was necessary to develop some mathematical relations involving the model to obtain the two constants needed for each material. The obtained results were satisfactory, making possible the realization of more detailed studies about the application of these mathematical relations, as much for the kaolin as for industrial minerals in general.
\end{abstract}

Keywords: Brightness, kaolin, Kubelka-Munk, contaminants. 
Teoria de Kubelka-Munk aplicada na indústria de minerais industriais: predição do teor de ...

\section{Introdução}

O valor econômico de diversos minerais industriais está diretamente associado às suas propriedades óticas. Isso se deve ao fato de que a maioria das indústrias consumidoras desses minerais industriais incorpora-os em produtos nos quais a aparência visual é controlada. Para essas indústrias, como, por exemplo, as de plásticos, papel, tintas e cerâmicos, variações na coloração das matérias-primas minerais implicam dificuldades no acerto da cor do produto final.

No caso particular do caolim, uma das maiores dificuldades no seu processamento é o controle da remoção dos seus contaminantes. Esses contaminantes são constituídos, principalmente, por óxidos de ferro e titânio e cada um responde de maneira diferenciada às operações unitárias de processamento.

Portanto o objetivo desse trabalho é a obtenção de fórmulas que possibilitem explorar as propriedades óticas do caolim e seus contaminantes (e minerais industriais em geral), de forma a otimizar as operações envolvidas na sua produção. Para tanto, foi explorada a teoria de Kubelka-Munk, que é largamente utilizada nas indústrias consumidoras de minerais industriais.

\subsection{Considerações teóricas}

A teoria de K-M prevê que, para uma camada de material de espessura $d$ sendo irradiada com um fluxo de radiação difusa monocromática na direção -X, representado por I, a relação entre este fluxo e aquele que está sendo espalhado de volta pelo material (na direção $+x$ ), representado por $J$, em uma fina camada de espessura $d x$ é dada pelo sistema de equações diferenciais (1):

$$
\begin{aligned}
& -\frac{d I}{d x}=-(K+S) I+S J \\
& \frac{d J}{d x}=-(K+S) J+S I
\end{aligned}
$$

Onde $K$ e $S$ representam, respectivamente, os coeficientes de absorção e de es- palhamento da luz por unidade de comprimento. Resolvendo o sistema (1), pode-se chegar à "Função de KubelkaMunk”, expressa na equação 2:

$$
\frac{K}{S}=\frac{\left(1-R_{\infty}\right)^{2}}{2 R_{\infty}} \equiv F\left(R_{\infty}\right)
$$

Onde $R_{\infty}$ representa a reflectância de uma camada de material totalmente opaca (ou seja, com uma espessura tal que a luz não a atravesse). No caso de uma mistura de materiais (como um minério contaminado ou uma carga composta por vários minerais, por exemplo), $F\left(R_{\alpha}\right)$ (daqui em diante denominado apenas por $F$ ), é dado por:

$F=\frac{\Sigma K_{i} c_{i}}{\Sigma S_{i} c_{i}}$

No caso de um substrato branco em excesso (com $K \approx 0$ ), contendo outros elementos em concentrações baixas (daqui em diante denominados “contaminantes”), conforme Kortüm (1969), a Eq. (3) pode ser simplificada para:

$F(\lambda)-F_{0}(\lambda)=K_{1}(\lambda) c_{1}+K_{2}(\lambda) c_{2}+\ldots$

Onde $F_{0}$ representa o $F\left(R_{\alpha}\right)$ do substrato puro. Os valores de $\mathrm{K}$ de cada contaminante são, então, obtidos através de misturas binárias de concentração conhecida dos contaminantes com o substrato, com um contaminante de cada vez, pelo cálculo da inclinação da reta $F$ x $C$ obtida na Figura 1.

Uma vez conhecido o $K$ de todos os materiais para todo o espectro visível, a determinação de concentrações desconhecidas de contaminantes consiste em selecionar comprimentos de onda convenientes e resolver um sistema de equações de ordem igual ao número de contaminantes na mistura. Entretanto, na prática, surgem alguns problemas:

- A simplificação supõe que o valor de S de todos os materiais é constante em todo o espectro visível, o que, na realidade, não ocorre.

- Pelo fato de se desconsiderar o denominador na eq. (3), para se chegar à eq. (4), o $K$ dos contaminantes fica relativo ao substrato escolhido, ou seja, se for escolhido outro substrato, o valor de $K$ obtido para os contaminantes será diferente.

- Além disso, a eq. (4) só mantém o seu comportamento linear para concentrações muito baixas de contaminantes.

Essas dificuldades levaram à busca por um método mais robusto e confiável que a simplificação apresentada anteriormente. Para tanto, considere-se a Eq. (3), no caso em que há apenas o substrato e um contaminante (que receberão, respectivamente, os índices 0 e 1). A equação pode, então, ser escrita da seguinte forma:

$$
F=\frac{K_{0} c_{0}+K_{1} c_{1}}{S_{0} c_{0}+S_{1} c_{1}}
$$
tém-se:

Reescrevendo $c_{0}=1-c_{1}$ e $c_{1} \equiv c$, ob-

$$
F=\frac{K_{0}+\left(K_{1}-K_{0}\right) \cdot c}{S_{0}+\left(S_{1}-S_{0}\right) \cdot c}
$$

Derivando a expressão (6), em relação a c, e aplicando a aproximação linear de Taylor em torno do ponto $c=0$, é possível obter:

$$
F=\frac{K_{0}}{S_{0}}+\frac{S_{0} K_{1}-K_{0} S_{1}}{S_{0}{ }^{2}} \cdot c
$$

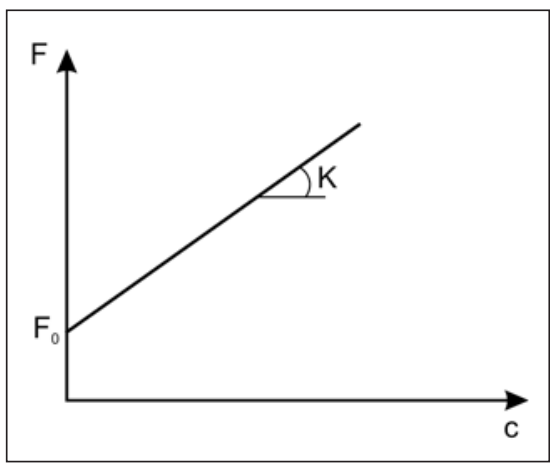

Figura 1 - Reta utilizada para o cálculo de K. 
Ítalo Gomes Gonçalves et al.

A expressão (7) representa uma reta semelhante àquela da Figura 1. No entanto, a inclinação da reta não é dada por um único valor e sim, por uma combinação das duas constantes, $K$ e $S$, dos dois materiais envolvidos. Isso elimina os problemas de "relatividade" entre contaminantes e substratos e não exige que S seja constante no espectro. Porém, agora a determinação de uma constante exige que se conheça outras três. A solução encontrada para este problema foi a utilização desta equação em conjunto com a teoria original de $K-M$, como será visto mais adiante.

\subsection{Considerações práticas}

De acordo com Kortüm (1969), a partir da reflectância de uma camada fina de material é possível obter a constante $\mathrm{S}$ do mesmo através da expressão:

$$
S=\frac{1}{d \cdot b} \operatorname{coth}^{-1}\left(\frac{1-a R_{0}}{b R_{0}}\right)
$$

Onde $d$ representa a espessura da camada, $a$ e $b$ são ambos funções de $R_{\infty}$ do material e $R_{0}$ é a reflectância medida para uma espessura d sobre um fundo não refletor. Conhecendo $R_{\infty}$ e $S$, é possível obter K através da eq. (2), ou seja:

$K=F . S$

A utilização da Eq. (8) pode ser difícil devido a problemas experimentais (o erro em $S$ tende a ser alto para altas reflectâncias) ou práticos (a obtenção de uma camada muito fina de um líquido ou pó, por exemplo), mas será necessária, no mínimo, para a obtenção de $K$ e $S$ do material a ser definido como substrato.

Conhecendo-se então, $K$ e $S$ do substrato, toma-se a inclinação da reta na Eq. (7), lembrando que o índice 0 representa o substrato, enquanto que o índice 1 representa o material cujas constantes são desconhecidas:

$$
F^{\mid}=\frac{S_{0} K_{1}-K_{0} S_{1}}{S_{0}^{2}}
$$

$$
\text { ou } F^{\mid} S_{0}^{2}=S_{0} K_{1}-K_{0} S_{1}
$$

Dividindo essa expressão por $S_{0} S_{1}$, obtém-se:

$\frac{F^{\mid} S_{0}}{S_{1}}=\frac{K_{1}}{S_{1}}-\frac{K_{0}}{S_{0}}$

Escrevendo $\frac{K_{0}}{S_{0}} \equiv F_{0}$ e $\frac{K_{1}}{S_{1}} \equiv F_{1}$ e organizando os termos, pode-se chegar à expressão:

$S_{1}=\frac{F^{\mid} S_{0}}{F_{1}-F_{0}}$

Ou seja, pode-se obter o coeficiente de espalhamento de um material desconhecido conhecendo-se $R_{\infty}$ (e por conseqüência $F$ ) de cada material puro, o coeficiente de espalhamento do substrato (obtido pela eq. (8)) e a inclinação da reta $F \times C$ para concentrações muito baixas do material desconhecido, obtida por uma regressão linear. E uma vez conhecido $S_{1}, K_{1}$ pode ser obtido através da expressão:

$K_{1}=F_{1} \cdot S_{1}$

Conhecidos os valores de $K$ e $S$ para todos os materiais, o objetivo agora é desenvolver um método para a determinação de concentrações a partir da medição de $F\left(R_{\infty}\right)$ de uma mistura. Para isso, considerando-se a Eq. (5) para um número qualquer de contaminantes e escrevendo $c_{0}=1-\sum c_{N}$ obtém-se:

$F=\frac{K_{0}+\left(K_{1}-K_{0}\right) c_{1}+\left(K_{2}-K_{0}\right) c_{2}+\ldots+\left(K_{n}-K_{0}\right) c_{n}}{S_{0}+\left(S_{1}-S_{0}\right) c_{1}+\left(S_{2}-S_{0}\right) c_{2}+\ldots+\left(S_{n}-S_{0}\right) c_{n}}$

Passando o denominador para o outro lado da igualdade e colocando em evidência $c_{1}, C_{2}$, etc., chega-se à expressão:

$\left[K_{1}-K_{0}-F\left(S_{1}-S_{0}\right)\right] c_{1}+\left[K_{2}-K_{0}-F\left(S_{2}-S_{0}\right)\right] c_{2}+\ldots+\left[K_{n}-K_{0}-F\left(S_{n}-S_{0}\right)\right] c_{n}=F S_{0}-K_{0}$

Escreve-se:

$$
\begin{aligned}
& {\left[K_{1}-K_{0}-F\left(S_{1}-S_{0}\right)\right] \equiv A(\lambda)} \\
& {\left[K_{2}-K_{0}-F\left(S_{2}-S_{0}\right)\right] \equiv B(\lambda)} \\
& \vdots \\
& {\left[K_{n}-K_{0}-F\left(S_{n}-S_{0}\right)\right] \equiv N(\lambda)} \\
& F S_{0}-K_{0} \equiv \gamma(\lambda)
\end{aligned}
$$

Pela representação em forma matricial, na qual cada linha representa um comprimento de onda, obtém-se:

$$
\left[\begin{array}{cccc}
A_{1} & B_{1} & \cdots & N_{1} \\
A_{2} & B_{2} & \cdots & N_{2} \\
A_{3} & B_{3} & \cdots & N_{3} \\
\vdots & \vdots & \vdots & \vdots \\
\vdots & \vdots & \vdots & \vdots \\
A_{n} & B_{n} & \cdots & N_{n}
\end{array}\right]\left[\begin{array}{c}
C_{1} \\
C_{2} \\
\vdots \\
C_{n}
\end{array}\right]=\left[\begin{array}{c}
\gamma_{1} \\
\gamma_{2} \\
\gamma_{3} \\
\vdots \\
\vdots \\
\gamma_{N}
\end{array}\right]
$$


Teoria de Kubelka-Munk aplicada na indústria de minerais industriais: predição do teor de ...

Aplicando-se o método dos mínimos quadrados de forma a utilizar todo o espectro medido, chega-se à expressão:

$\left[\begin{array}{cccc}\Sigma A_{i}^{2} & \Sigma A_{i} B_{i} & \cdots & \Sigma A_{i} N_{i} \\ \Sigma A_{i} B_{i} & \Sigma B_{i}^{2} & \cdots & \Sigma B_{i} N_{i} \\ \vdots & \vdots & \ddots & \vdots \\ \Sigma A_{i} N_{i} & \Sigma B_{i} N_{i} & \cdots & \Sigma N_{i}^{2}\end{array}\right]\left[\begin{array}{c}c_{1} \\ c_{2} \\ \vdots \\ c_{n}\end{array}\right]=\left[\begin{array}{c}\Sigma A_{i} \gamma_{i} \\ \Sigma B_{i} \gamma_{i} \\ \vdots \\ \Sigma N_{i} \gamma_{i}\end{array}\right]$

A expressão (16) representa um sistema de equações lineares de ordem igual ao número de materiais envolvidos na mistura menos um. A aplicação dessas fórmulas, para o caso do caolim, está descrita a seguir.

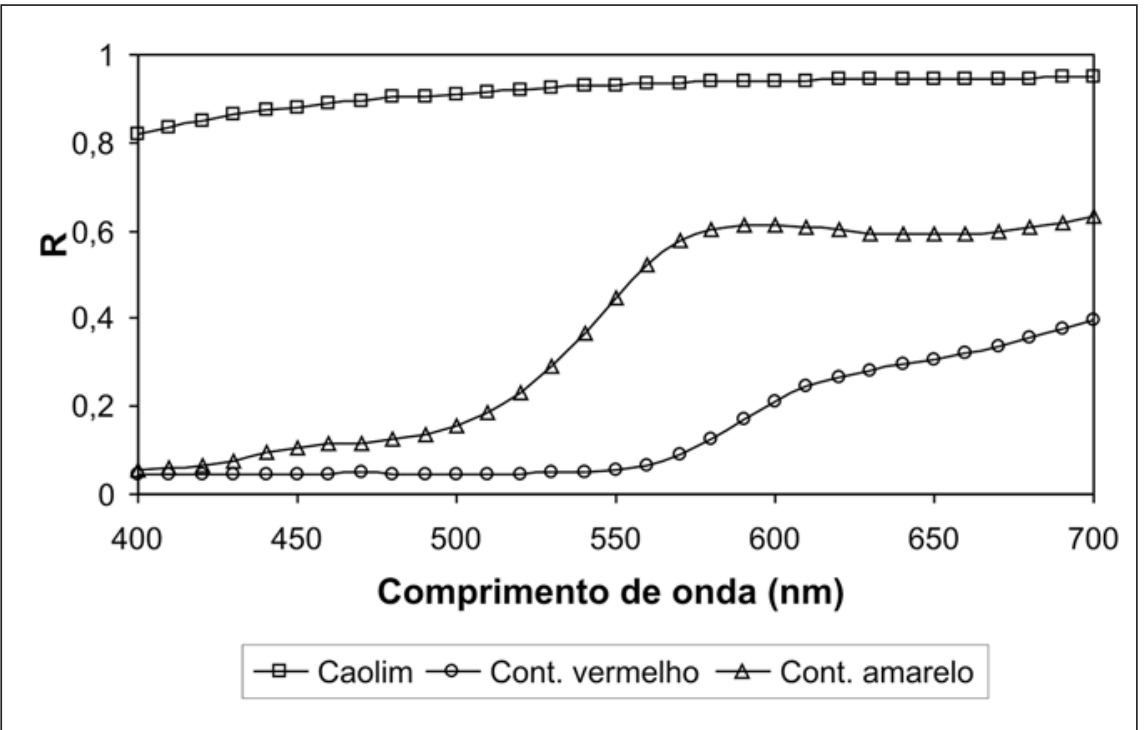

Figura 2 - Espectro de reflectância dos materiais estudados.

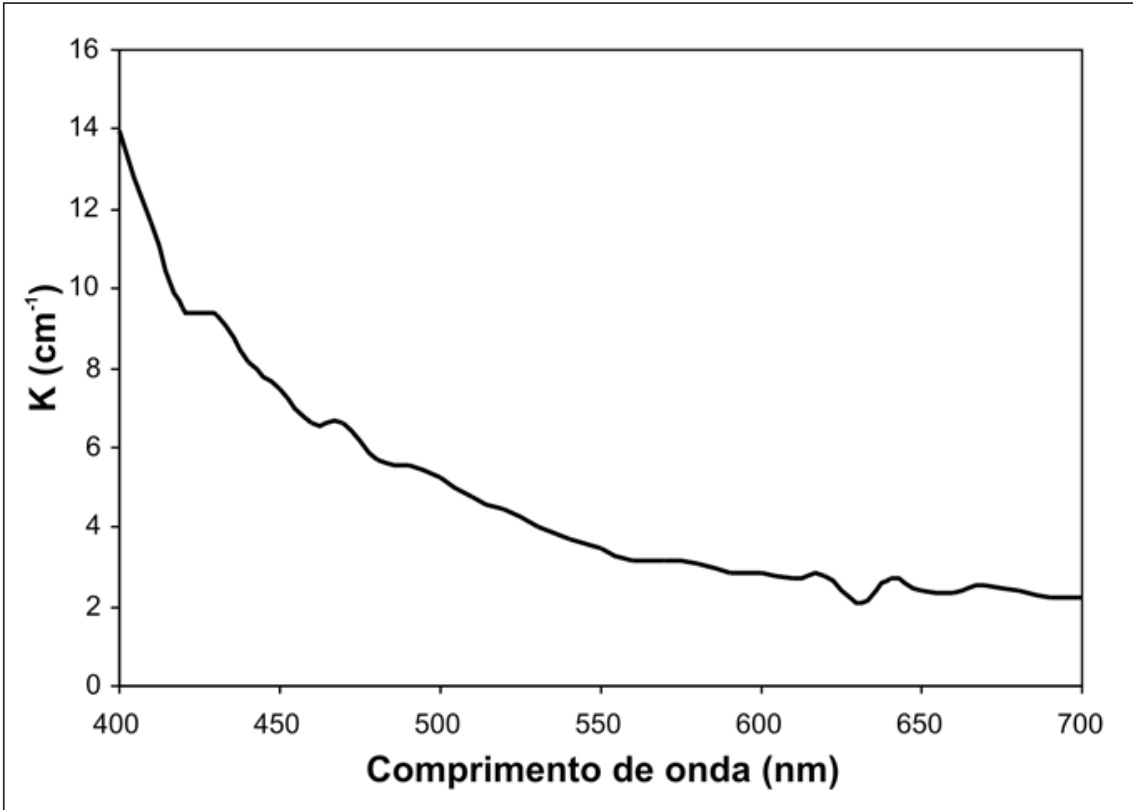

Figura 3 - Espectro de $K$ do caolim.

\section{Materiais e métodos}

Os principais contaminantes encontrados no caolim são os óxidos, tais como hematita, goethita, rutilo, etc. Pela necessidade de se possuírem todos os materiais envolvidos puros, utilizou-se um caolim beneficiado, livre de impurezas e pigmentos artificiais à base de óxidos de ferro (um de cor vermelha e outro, amarela), de forma a simular um caolim contaminado. Os espectros de $R_{\infty}$ dos materiais estão apresentados na Figura 2.

A reflectância dos materiais foi medida através de pastilhas. As pastilhas são preparadas com o auxílio de uma prensa, que compacta o pó confinado em um anel metálico em contato com uma superfície de vidro. A medição da reflectância é feita sobre a superfície da pastilha que estava em contato com o vidro. Em todas as medidas, foi utilizado um espectrofotômetro Minolta modelo 2600d.

No caso de uma camada fina, necessária para a utilização da eq. (8), o material foi prensado manualmente contra uma placa preta de plástico com o auxílio de uma lamínula de plástico e a espessura da camada foi medida com um micrômetro digital Mituoyo IP65.

\section{Resultados e discussão}

O primeiro passo foi a determinação das constantes $K$ e $S$ do caolim, através das equações (8) e (9). Os resultados encontram-se nas Figuras 3 e 4 . Supostamente, essas curvas deveriam ser mais suaves do que as curvas realmente obtidas. Esse ruído pode ser devido a erros experimentais, pois, para a menor espessura de camada que foi possível ser obtida (da ordem de $0,15 \mathrm{~mm}$ ), o valor de $R_{0}$ medido estava muito próximo do $R_{\infty}$ do caolim, caso em que a eq. (8) tende a gerar resultados menos exatos. 
Ítalo Gomes Gonçalves et al.

O segundo passo foi utilizar as Eqs. (11) e (12) para descobrir as constantes dos contaminantes. Para a obtenção da reta da Eq. (7), foram feitas misturas com concentrações de até $0,1 \%$ em massa. Em todos os comprimentos de onda medidos foram obtidos coeficientes de correlação maiores que 0,95 , conforme a Figura 5. Os espectros de K e S obtidos para os contaminantes estão apresentados nas Figuras 6 e 7 .

Para o presente caso, o sistema em (16) foi resolvido para os dois contaminantes apresentados, denominando-se o caolim como substrato. Isso levou a um sistema de equações 2 x2. Os experimentos realizados e o erro obtido são mostrados na Tabela 1.

\section{Conclusões}

1. As fórmulas aqui apresentadas se mostram bem sucedidas como um passo na direção de uma ferramenta de otimização de processos para o caolim, tendo como base suas propriedades óticas.

2. O erro experimental obtido pode ser reduzido ao se descobrir uma maneira de se obter uma camada de caolim mais fina, com espessura e reflectância possíveis de serem medidas com menor erro.

3. A teoria de Kubelka-Munk tem potencial para ser aplicada também para outros minerais industriais, tanto como uma ferramenta de otimização de processo, como de planejamento de lavra. A maior dificuldade se encontra na necessidade de obtenção de amostras puras de todos os minerais envolvidos.

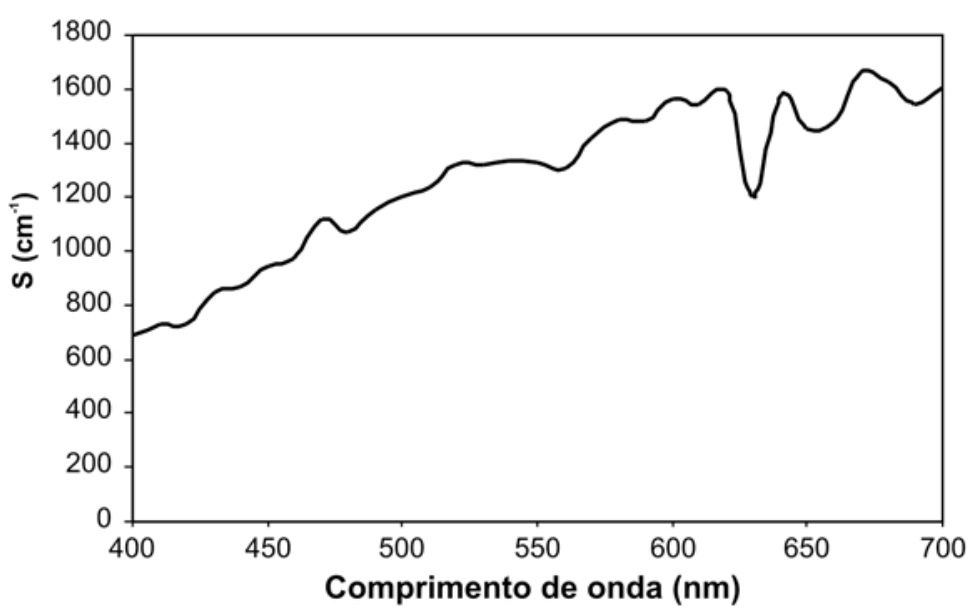

Figura 4 - Espectro de $S$ do caolim.

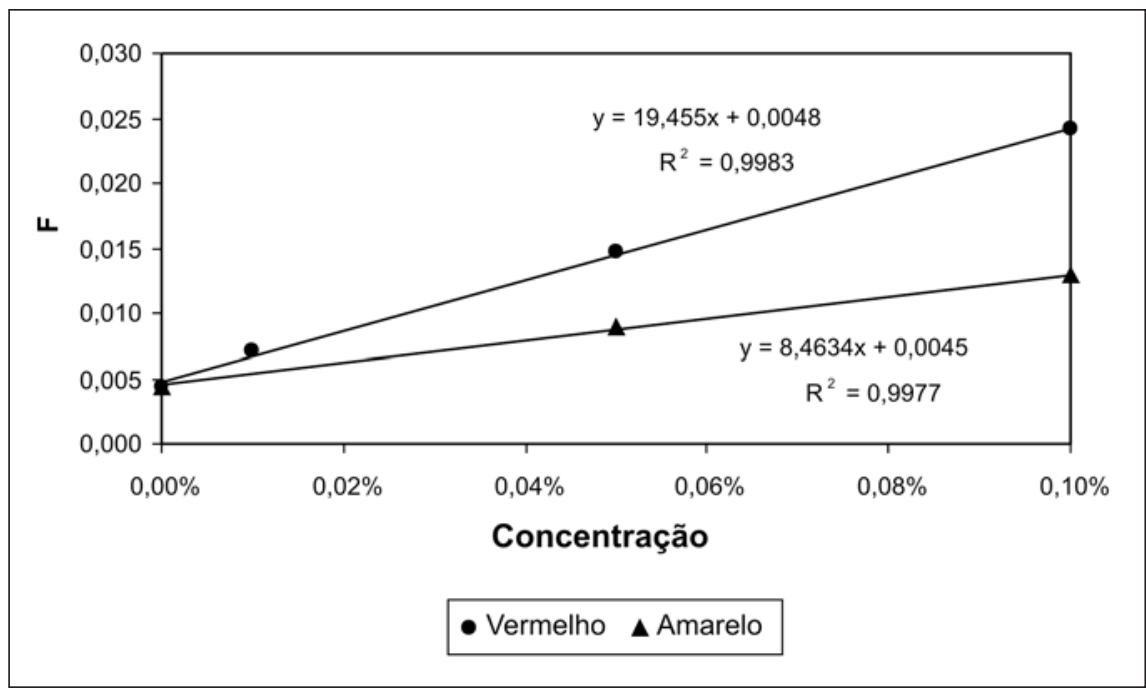

Figura 5 - Contaminantes em baixas concentrações para aplicação da eq. (11). Comprimento de onda: $500 \mathrm{~nm}$.

Tabela 1 - Concentrações reais e calculadas. Erro absoluto médio: vermelho - 0,004\%, amarelo $-0,009 \%$.

\begin{tabular}{c|c|c|c|c}
\hline \multirow{2}{*}{ Ensaio } & \multicolumn{2}{|c|}{ Conc. Vermelho } & \multicolumn{2}{c}{ Conc. Amarelo } \\
\cline { 2 - 5 } & Real & Calculado & Real & Calculado \\
\hline 1 & $0.020 \%$ & $0.020 \%$ & $0.080 \%$ & $0.075 \%$ \\
\hline 2 & $0.040 \%$ & $0.043 \%$ & $0.040 \%$ & $0.039 \%$ \\
\hline 3 & $0.070 \%$ & $0.072 \%$ & $0.020 \%$ & $0.021 \%$ \\
\hline 4 & $0.050 \%$ & $0.054 \%$ & $0.000 \%$ & $-0.001 \%$ \\
\hline 5 & $0.020 \%$ & $0.018 \%$ & $0.080 \%$ & $0.059 \%$ \\
\hline 6 & $0.080 \%$ & $0.071 \%$ & $0.020 \%$ & $0.008 \%$ \\
\hline 7 & $0.080 \%$ & $0.070 \%$ & $0.080 \%$ & $0.060 \%$ \\
\hline
\end{tabular}


Teoria de Kubelka-Munk aplicada na indústria de minerais industriais: predição do teor de ...

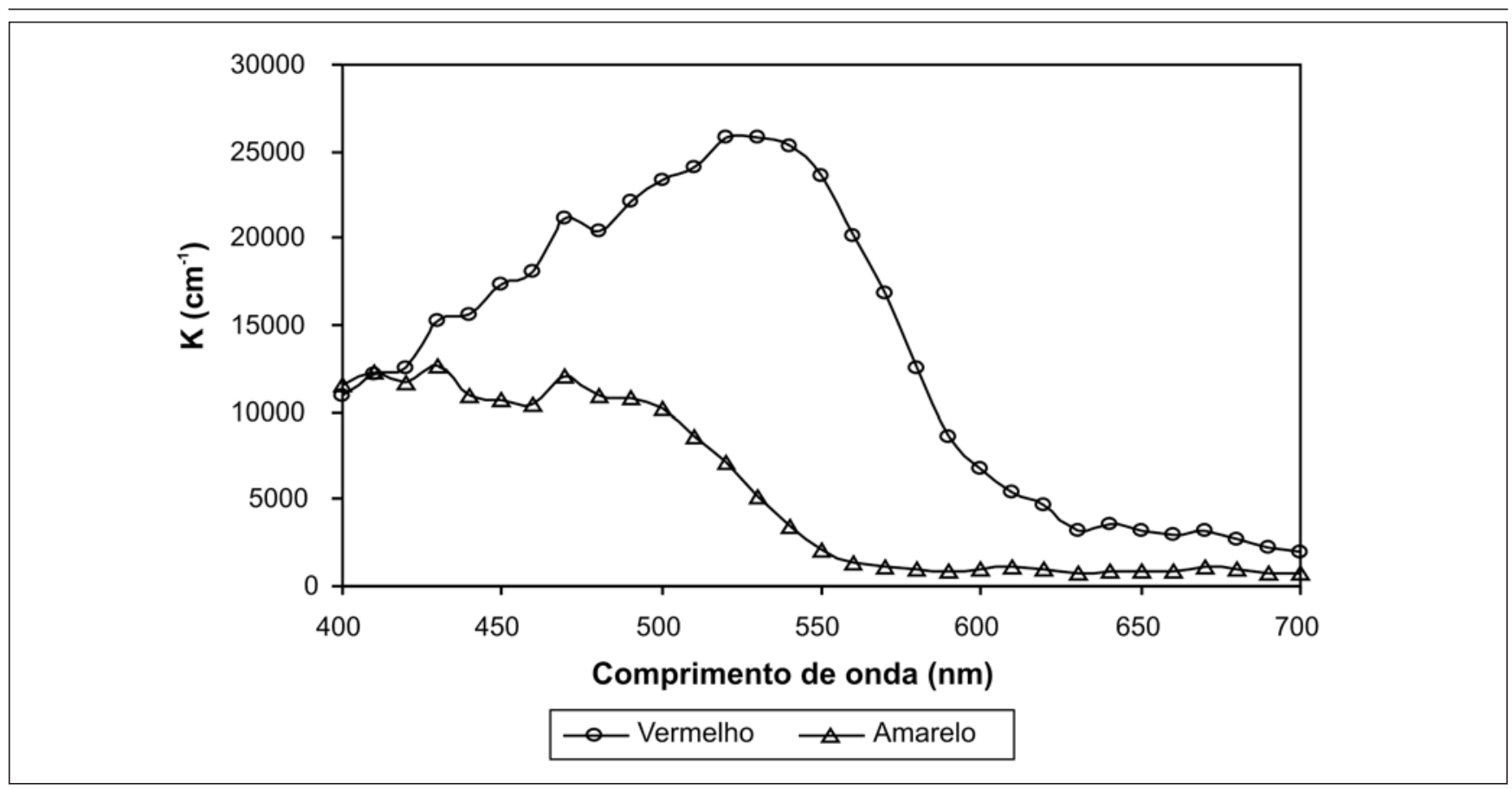

Figura 6 - Espectro de $\mathrm{K}$ dos contaminantes.

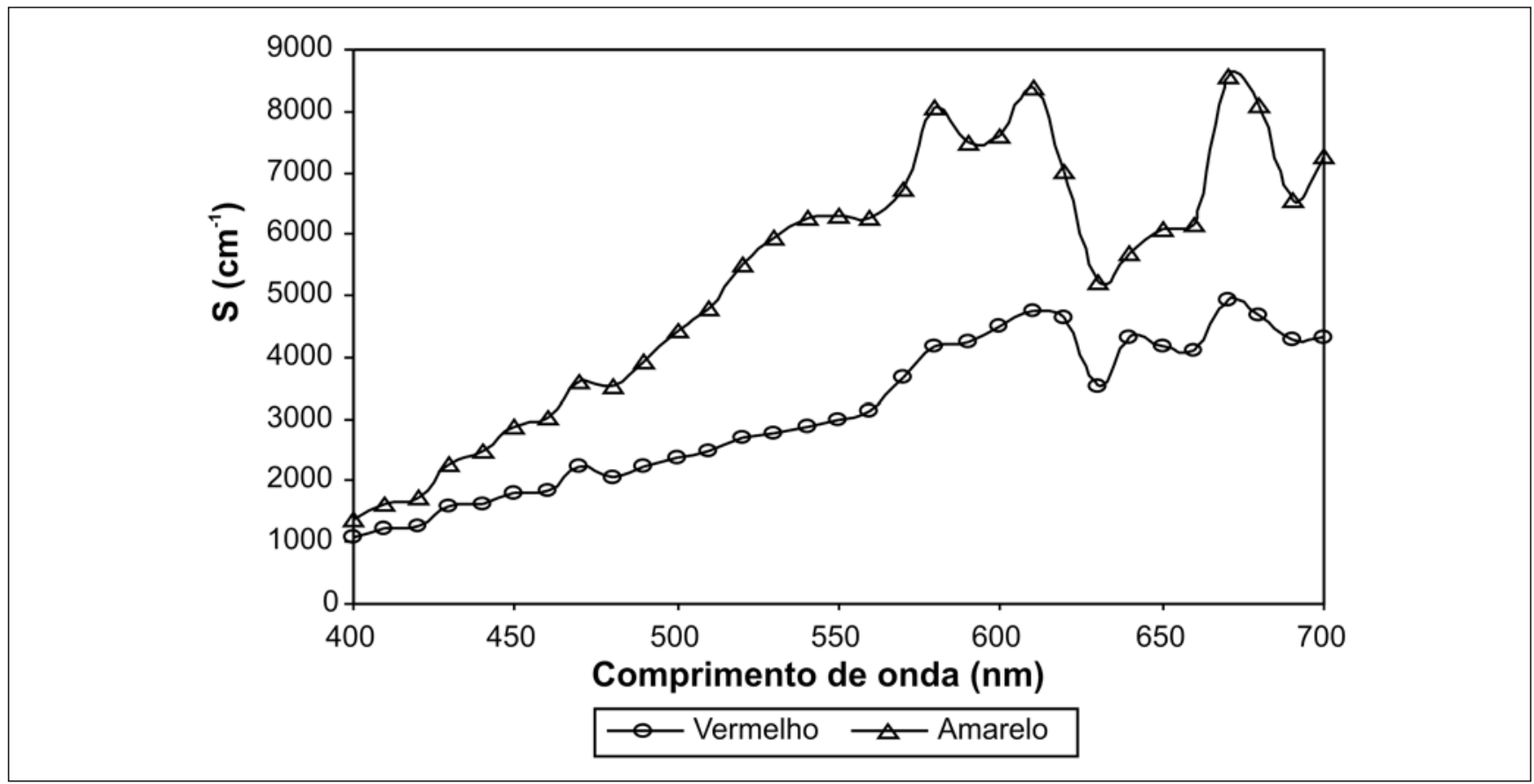

Figura 7 - Espectro de S dos contaminantes.

\section{Referências bibliográficas}

KORTÜM, G. Reflectance spectroscopy. Principles, methods, applications. Springer-Verlag, 1969.

PETTER, C.O. Contribution à l'etude de la valorisation de kaolins pour l'industrie papetiere: mise au point d'une methodologie colorimetrique, application à la selectivité miniere. École des Mines de Paris, 1994. (These).

PETTER, C.O., BATISTON, E.L., GONÇALVES, I.G., PEIXOTO, C.A. Determinação do teor de contaminantes colorantes no caolim a partir do espectro de reflectância difusa. In: ENTMME, 21, 2005. Natal. Anais... Natal: O2, 2005. v 2, p. 490-495.

Artigo recebido em 26/12/2006 e aprovado em 13/03/2007. 